\title{
Spin: The Effects of Acute Exercise on Speech Perception
}

\author{
Lakyn Kearns, Ashley Rich, Natalie Pita, Kayoko Okada*
}

Department of Psychology, Loyola Marymount University, Los Angeles, USA

Email address:

kayoko.okada@lmu.edu (K. Okada)

${ }^{*}$ Corresponding author

To cite this article:

Lakyn Kearns, Ashley Rich, Natalie Pita, Kayoko Okada. Spin: The Effects of Acute Exercise on Speech Perception. Psychology and Behavioral Sciences. Vol. 8, No. 3, 2019, pp. 67-71. doi: 10.11648/j.pbs.20190803.12

Received: April 8, 2019; Accepted: May 20, 2019; Published: June 26, 2019

\begin{abstract}
Previous research suggests that adults who exercise regularly perform better on cognitive tasks. Most of these studies have focused on the benefits of aerobic exercise on executive function in the aging population. Very few studies to date have focused on how exercise affects perceptual abilities, particularly in healthy young adults. This is particularly important since poor cardiovascular health and low fitness levels are risk factors for hearing loss. The purpose of this study was to test whether exercising, defined as cycling for 30 minutes, affects auditory perception. College-aged participants performed a speech perception task before and after 30 minutes of aerobic exercise on a stationary bicycle. Auditory stimuli were speech that varied in intelligibility and have been used in previous experiments. Words were presented in noise and participants reported aloud the word they identified. The experimenter recorded participant responses during the experiment and the percentage of words correctly identified was calculated. Analysis revealed that there was a significant difference between the percentage of words correctly identified before exercise and after exercise. These results suggest that acute aerobic exercise has immediate effects that improve speech perception ability.
\end{abstract}

Keywords: Exercise, Speech Perception, Auditory Processing, Hearing

\section{Introduction}

Effective communication impacts our social, emotional, and intellectual well-being as individuals. Speech perception is one way in which humans communicate. There is an agerelated disabling hearing loss that affects approximately $25 \%$ of adults over the age of 64 and this rate increases to $50 \%$ for adults over the age of 75 [1]. Despite research examining the relationship between the aging brain and cognitive performance, it remains unclear to what extent exercise slows the progression of age related decline in perceptual function.

In 1975, Spirduso published research showing that active older adults exhibit better cognitive performance than inactive older adults [2]. Since then, numerous experiments have explored the effects of aerobic exercise on cognitive function. Many of these experiments have found that there is a positive relationship between acute bouts of exercise and performance on memory and executive function tasks [3-5]. For example, studies show that aerobic exercise improves speed in cognitive controls tasks, improves short-term and working memory function, and improves reaction time in attention tasks. Research also suggests that aerobic exercise improves memory retention and plays a protective role against dementia and Alzheimer's Disease [6-8].

With the advent of neuroimaging, recent studies have focused on understanding the neuroanatomical changes that underlie the differences in cognitive performance [9-11]. For example, studies have shown that aerobic exercise increases gray matter volume in the hippocampus, a sub-cortical structure critical for memory function [10]. Other studies have shown an increase in gray matter volume in frontal lobe regions that support executive functioning [9, 12], and greater white matter connectivity across brain regions [9].

Much of the research to date has focused on examining the benefits of exercise on the aging brain and its relationship to cognitive functioning. Fewer studies have examined the benefits of exercise on healthy young people, and the existing literature reports conflicting findings. For example, one study examined short term memory performance and set switching ability in young adults and found no benefit for either task after 40 minutes of cycling [13]. The same study reported a small benefit in a delayed memory task. Another study that 
compared memory-search performance after aerobic vs. anaerobic training in healthy men (mean age 43 years) did not find any benefits of aerobic exercise [6]. They found that participants' initial level of fitness was positively correlated with memory performance. Finally, there are reports that over-exercise can have detrimental effects on memory performance [14].

Other studies with young adults have reported significant benefits of exercise on cognitive functioning [15]. For example, Chang, Chi, Etnier, Wang, Chu and Zhou [16] used a Stroop task to measure cognitive performance in college aged participants and found that executive function was enhanced after 20 minutes of exercise. In a longitudinal study conducted on Swedish men, researchers found that cardiovascular fitness at age 15 predicted cognitive performance at 18 years old as well as educational achievements later in life [15]. Some other studies have shown that fitness is positively correlated with better executive control performance $[17,18]$.

Although there is growing interest in understanding the role of exercise in supporting cognitive functioning, particularly in relation to aging, there is a dearth of studies examining the relationship between exercise and perceptual abilities. One study examined first year medical students' reaction times to auditory and visual stimuli and found that students who exercised regularly had significantly faster reaction times compared to sedentary students in both tasks [19]. Another study demonstrated that aerobic exercise improved visual attention and visuospatial abilities in college students [20]. Of the studies examining perceptual abilities, most have focused on understanding visual perceptual abilities, not auditory perceptual abilities. Given the link between cardiovascular health and hearing loss, this seems particularly important.

Our study investigates the immediate effects of aerobic exercise on auditory perception in college students. Participants were presented with speech that varied in intelligibility [21] and they were asked to identify words presented in noise. We hypothesize that aerobic exercise, defined as 30 minutes of cycling on a stationary bicycle, will increase the ability to correctly identify words in the auditory perception task.

\section{Method}

\subsection{Participants}

Twenty-five participants between 18 and 21 years of age (17 females and 8 males) were recruited from the Loyola Marymount University (LMU) Human Subject Pool and were given course credit for participation. Informed consent was obtained from each subject as required by the LMU Institutional Review Board. Participants also completed the Physical Activity Readiness Questionnaire (PAR-Q) developed by the National Academy of Sports Medicine to ensure that they were able to engage in physical activity. All participants reported to be in good health and reported no hearing impairments.

\subsection{Stimuli}

Stimuli were 120 words that varied in intelligibility and have been used in previously published speech perception experiments $[21,22]$. The words were recorded by a male speaker and consisted of monosyllabic consonant-vowelconsonant words controlled for word frequency, neighborhood density and phonotactic frequency (see [21] for specific metrics). Each of the words was high pass filtered $(200 \mathrm{~Hz})$ and low pass filtered to create four conditions that varied in intelligibility. Low pass cut-offs were $400 \mathrm{~Hz}, 1000 \mathrm{~Hz}, 1600 \mathrm{~Hz}$ and $3150 \mathrm{~Hz}$. These cut-offs produced speech with varying levels of intelligibility, with words low-passed at $400 \mathrm{~Hz}$ creating speech that is least intelligible, and words low-passed at $3150 \mathrm{~Hz}$ creating words most intelligible. From the list of 120 words, 60 were selected to use during the pre-exercise testing session (session 1) and 60 words were presented during the postexercise testing session (session 2). Fifteen words from each of the four intelligibility conditions were selected randomly to form the 60 words. Words were repeated twice to form a total of 120 trials presented in each session. As in Vaden, Kuchinsky, Keren, Harris, Ahlstrom, Dubno and Eckert [21] none of the words were repeated across intelligibility conditions to prevent memory and priming effects. Word lists were randomized for each participant, and words that were presented during session 1 were not presented during session 2. Words were presented at $75 \mathrm{~dB}$ SPL and played against spectral-shaped noise that was digitally generated and played continuously throughout the session at $65 \mathrm{db}$ SPL. Stimuli were controlled using Cogent Software running on Matlab.

\subsection{Procedure}

The experiment was completed in two sessions. In the first session (pre-exercise session), participants gave informed consent to participate in the study and completed a demographics and exercise questionnaires. Participants were then given a PAR-Q form to certify that they were able to participate in the study. After forms were completed, participants were seated in front of the computer and instructed on the task. They were instructed to listen to the auditory stimuli and report aloud the word they heard. The experiment started with a short practice session that consisted of 16 words. This was followed by 120 words presented in noise. On each trial, a white fixation appeared on screen against a black background to cue the subject that a word was going to be presented. This was followed by a single word played against noise. The experimenter recorded participant responses and errors during the experiment. The study was self-paced and participants hit the space bar to advance to the next trial after providing a verbal response. Stimuli were played over headphones and controlled using Cogent Software running on Matlab (Mathworks).

After the experiment, participants were scheduled for a second session and were instructed to wear exercise clothes and athletic shoes. The two testing sessions were separated by a minimum of 1 week. The second session started with 
measurement of subject's aerobic heart rate zone. The aerobic zone was considered $70 \%-80 \%$ of the participant's maximum heart rate, and was calculated using each subject's age and maximum heart rate $(\mathrm{MHR}=208-(0.7 *$ age $))$. Subjects were informed of their target zones before beginning exercise and were informed to remain in these zones during the spin session. Research assistants monitored participant's heart rate throughout the cycling period using a Garmin Forerunner Multisport Watch and Garmin premium heart rate monitor chest strap.

For the second session, subjects exercised for 30 minutes accompanied by a music playlist created for this study. The exercise session was modeled after similar experiments [23] and consisted of a five-minute cycling warm up, followed by 20-minutes of cycling while in their aerobic heart rate zone (calculated as $70-80 \%$ of maximum heart rate), and a fiveminute cool down. Participants were then given five minutes to rest before completing the speech task. The procedure for the speech task was the same as the first session. None of the stimuli used in the first session was used in the second session, and the two testing session were separated by at least 1 week interval. Following the completion of the experiment, subjects were debriefed.

\section{Results}

For each participant and each perceptual category, the number of words correctly identified pre-exercise was subtracted from the number of words correctly identified post-exercise. These difference scores were entered into a repeated measures one-way ANOVA. The analysis revealed that there was a significant difference between the four frequency levels $F(3,72)=7.05$, $p<.001$. In addition, post-hoc tests revealed a significant difference between the $400 \mathrm{~Hz}$ and $3150 \mathrm{~Hz}$ levels $(p=.01)$ and between the $1000 \mathrm{~Hz}$ and $3150 \mathrm{~Hz}$ levels ( $p=.026)$. Table 1 shows the mean number of words identified in each of the four categories before and after exercise. Figure 1 illustrates the difference scores across the four categories.

Table 1. Mean number of words identified in each of the four intelligibility conditions.

\begin{tabular}{lllll}
\hline & $\mathbf{4 0 0} \mathbf{~ H z}$ & $\mathbf{1 0 0 0 ~} \mathbf{~ H z}$ & $\mathbf{1 6 0 0 ~} \mathbf{~ z z}$ & $\mathbf{3 1 5 0 ~} \mathbf{~ z z}$ \\
\hline Pre-exercise & 0.76 & 4.24 & 9.12 & 11.28 \\
Post-exercise & 0.52 & 4.44 & 9.76 & 15.32 \\
\hline
\end{tabular}

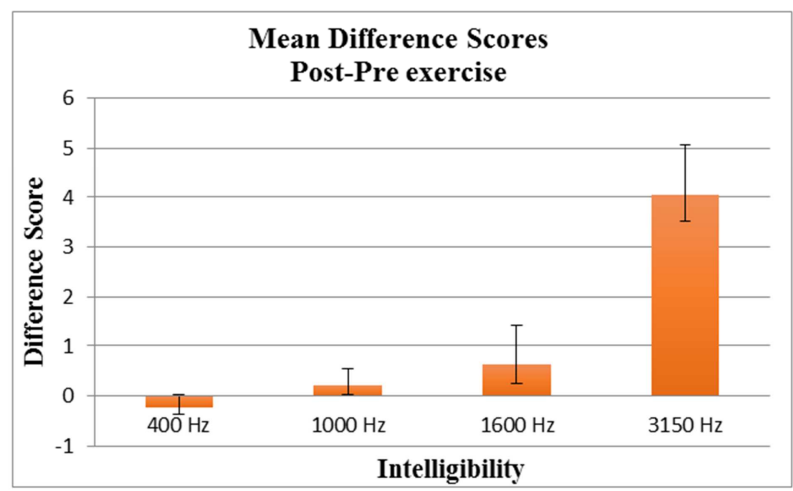

Figure 1. Mean difference between post-exercise identification scores and pre-exercise identification scores for each of the intelligibility conditions.
Finally, the four frequency groups were collapsed to test if participants were able to identify a higher percentage of the words after exercise. A paired samples t-test revealed that there was a significant difference in the number of words correctly identified during session one $(\mathrm{M}=25.4)$ and session two $(\mathrm{M}=29.83), t(24)=-3.97, p=.001$. Participants identified significantly more words after aerobic exercise.

\section{Discussion}

As hypothesized, 30 minutes of exercise on a stationary bicycle resulted in an increase in the ability of participants to identify words embedded in noise. This indicates that aerobic exercise can enhance auditory perception. Comparing pre and post test scores for each frequency filter condition, we found that participants were able to correctly identify a higher number of more intelligible words after exercise. That is, the largest benefit of exercise was observed in the $3150 \mathrm{~Hz}$ condition. We believe this is the first demonstration that auditory perceptual function is enhanced by acute aerobic exercise.

This is an important finding because age-related hearing loss affects half of aging population over the age of 75 . High blood pressure and cardiovascular disease are risk factors for age-related decline in auditory function and hearing loss [2427]. In a cross-sectional study, Hutchinson, Alessio and $R$ Baiduc [27] examined 101 people between the age of 10-78 years and they found that older adults (over 49) had worse hearing than younger people. Importantly, they found that older adults with high cardiovascular health had better hearing compared to older adults with low cardiovascular health. Poor cardiovascular health and poor circulation cause damage to hair cells in the cochlea and this causes hearing loss. This suggests that improving heart health is beneficial in slowing hearing loss and aerobic exercise can improve overall cardiovascular health.

\section{Conclusion}

Research demonstrates that there are tremendous benefits of aerobic exercise on physical and mental health across the lifespan [28]. A healthy lifestyle that includes regular physical exercise leads to decreased risk for disease (e.g., heart disease, diabetes, stroke, obesity), improved mood regulation in patients with mood disorders, increased concentration, improvement in memory functioning, and improvement in executive function. Our work contributes to this growing body of work by demonstrating that aerobic exercise enhances auditory functioning.

We recognize that our study only investigates the immediate effects of acute exercise on auditory perception, and our work cannot directly address the benefits of longterm exercise on auditory functioning in aging. However, given the lack of research in this domain, we believe that our study with college-aged population is an important step to stimulate more research in this area. Future work should examine the effects of long-term exercise and fitness levels on aging and hearing. 


\section{Conflict of Interest Statement}

The authors declare that they have no competing interests.

\section{References}

[1] NIH. "Quick Statistes About Hearing," https://www.nidcd.nih.gov/health/statistics/quick-statisticshearing -6 .

[2] W. W. Spirduso, "Reaction and Movement Time as a Function of age and Physical Activity Level1," Journal of Gerontology, vol. 30, no. 4, pp. 435-440, 1975.

[3] H. van Praag, "Exercise and the brain: something to chew on," Trends in neurosciences, vol. 32, no. 5, pp. 283-290, 2009.

[4] S. Colcombe, and A. F. Kramer, "Fitness Effects on the Cognitive Function of Older Adults: A Meta-Analytic Study," Psychological Science, vol. 14, no. 2, pp. 125-130, 2003/03/01, 2003.

[5] Y. K. Chang, J. D. Labban, J. I. Gapin, and J. L. Etnier, “The effects of acute exercise on cognitive performance: A metaanalysis," Brain Research, vol. 1453, pp. 87-101, 2012/05/09/, 2012.

[6] J. A. Blumenthal, and D. J. Madden, "Effects of aerobic exercise training, age, and physical fitness on memory-search performance," Psychology and Aging, vol. 3, no. 3, pp. 280$285,1988$.

[7] J. E. Ahlskog, Y. E. Geda, N. R. Graff-Radford, and R. C. Petersen, "Physical exercise as a preventive or diseasemodifying treatment of dementia and brain aging," Mayo Clinic proceedings, vol. 86, no. 9, pp. 876-884, 2011.

[8] S. P. Cass, "Alzheimer's Disease and Exercise: A Literature Review," Current Sports Medicine Reports, vol. 16, no. 1, pp. 19-22, 2017.

[9] A. F. Kramer, D. X. Marquez, E. McAuley, J. S. Kim, K. I. Erickson, L. Hu, P. E. Scalf, R. Prakash, S. J. Colcombe, and S. Elavsky, "Aerobic Exercise Training Increases Brain Volume in Aging Humans," The Journals of Gerontology: Series A, vol. 61, no. 11, pp. 1166-1170, 2006.

[10] K. I. Erickson, M. W. Voss, R. S. Prakash, C. Basak, A. Szabo, L. Chaddock, J. S. Kim, S. Heo, H. Alves, S. M. White, T. R. Wojcicki, E. Mailey, V. J. Vieira, S. A. Martin, B. D. Pence, J. A. Woods, E. McAuley, and A. F. Kramer, "Exercise training increases size of hippocampus and improves memory," Proceedings of the National Academy of Sciences of the United States of America, vol. 108, no. 7, pp. 3017-3022, 01/31, 2011.

[11] F. Gomez-Pinilla, and C. Hillman, "The influence of exercise on cognitive abilities," Comprehensive Physiology, vol. 3, no. 1, pp. 403-428, 2013.

[12] L. Chaddock-Heyman, K. I. Erickson, M. W. Voss, A. M. Knecht, M. B. Pontifex, D. M. Castelli, C. H. Hillman, and A. F. Kramer, "The effects of physical activity on functional MRI activation associated with cognitive control in children: a randomized controlled intervention," Frontiers in Human Neuroscience, vol. $\quad 7$, pp. 72, 03/1201/16/received02/25/accepted, 2013.

[13] P. D. Tomporowski, "Effects of acute exercise on executive processing, short-term and long-term memory AU - Coles, Kathryn," Journal of Sports Sciences, vol. 26, no. 3, pp. 333344, 2008/02/01, 2008.

[14] P. D. Tomporowski, "Effects of acute bouts of exercise on cognition," Acta Psychologica, vol. 112, no. 3, pp. 297-324, 2003/03/01/, 2003.

[15] M. A. I. Åberg, N. L. Pedersen, K. Torén, M. Svartengren, B. Bäckstrand, T. Johnsson, C. M. Cooper-Kuhn, N. D. Åberg, M. Nilsson, and H. G. Kuhn, "Cardiovascular fitness is associated with cognition in young adulthood," Proceedings of the National Academy of Sciences, vol. 106, no. 49, pp. 20906, 2009.

[16] Y. K. Chang, L. Chi, J. L. Etnier, C.-C. Wang, C.-H. Chu, and C. Zhou, "Effect of acute aerobic exercise on cognitive performance: Role of cardiovascular fitness," Psychology of Sport and Exercise, vol. 15, no. 5, pp. 464-470, 2014/09/01/, 2014.

[17] C. H. Hillman, D. M. Castelli, and S. M. Buck, "Aerobic Fitness and Neurocognitive Function in Healthy Preadolescent Children," Medicine \& Science in Sports \& Exercise, vol. 37, no. 11, pp. 1967-1974, 2005.

[18] J. R. Themanson, M. B. Pontifex, and C. H. Hillman, "Fitness and action monitoring: Evidence for improved cognitive flexibility in young adults," Neuroscience, vol. 157, no. 2, pp. 319-328, 2008/11/19/, 2008.

[19] A. Jain, R. Bansal, A. Kumar, and K. Singh, "A comparative study of visual and auditory reaction times on the basis of gender and physical activity levels of medical first year students," International Journal of Applied and Basic Medical Research, vol. 5, no. 2, pp. 124-127, May 1, 2015, 2015.

[20] C. H. Hillman, E. M. Snook, and G. J. Jerome, "Acute cardiovascular exercise and executive control function," International Journal of Psychophysiology, vol. 48, no. 3, pp. 307-314, 2003/06/01/, 2003.

[21] K. I. Vaden, S. E. Kuchinsky, N. I. Keren, K. C. Harris, J. B. Ahlstrom, J. R. Dubno, and M. A. Eckert, "Inferior frontal sensitivity to common speech sounds is amplified by increasing word intelligibility," Neuropsychologia, vol. 49, no. 13, pp. 3563-3572, 2011/11/01/, 2011.

[22] D. Dirks, S. Takayanagi, A. Moshfegh, P. Douglas Noffsinger, and S. A. Fausti, Examination of the Neighborhood Activation Theory in Normal and Hearing-Impaired Listeners, 2001.

[23] Y.-K. Chang, L. Chi, J. L. Etnier, C.-C. Wang, C.-H. Chu, and C. Zhou, "Effect of acute aerobic exercise on cognitive performance: Role of cardiovascular fitness," Psychology of Sport and Exercise, vol. 15, no. 5, pp. 464-470, 2014/09/01/, 2014.

[24] L. Brant, S. Gordon-Salant, J. D Pearson, L. L Klein, C. Morrell, E. J Metter, and J. L Fozard, Risk factors related to age-associated hearing loss in speech frequencies, 1996.

[25] K. Wattamwar, Z. J. Qian, J. Otter, M. J. Leskowitz, F. F. Caruana, B. Siedlecki, J. B. Spitzer, and A. K. Lalwani, "Association of Cardiovascular Comorbidities With Hearing Loss in the Older OldAssociation of Cardiovascular Comorbidities With Hearing Loss in the Older OldAssociation of Cardiovascular Comorbidities With Hearing Loss in the Older Old," JAMA Otolaryngology-Head \& Neck Surgery, vol. 144, no. 7, pp. 623-629, 2018. 
[26] R. H. Hull, and S. R. Kerschen, "The Influence of Cardiovascular Health on Peripheral and Central Auditory Function in Adults: A Research Review," American Journal of Audiology, vol. 19, no. 1, pp. 9-16, 2010.

[27] K. Hutchinson, H. Alessio, and R. R Baiduc, Association Between Cardiovascular Health and Hearing Function: Pure-
Tone and Distortion Product Otoacoustic Emission Measures, 2010.

[28] C. H. Hillman, K. I. Erickson, and A. F. Kramer, "Be smart, exercise your heart: exercise effects on brain and cognition," Nat Rev Neurosci, vol. 9, no. 1, pp. 58-65, 01//print, 2008. 